\title{
BMJ Open Qualitative study of antibiotic prescription patterns and associated drivers in Sudan, Guinea-Bissau, Central African Republic and Democratic Republic of Congo
}

\author{
Tonka Eibs, ${ }_{1}^{1}$ Alena Koscalova (10 , ${ }^{1}$ Mohit Nair, ${ }^{1}$ Paul Grohma, ${ }^{2}$ Gisa Kohler, ${ }^{1}$ \\ Rawa Gafar Bakhit, ${ }^{1}$ Mzia Thurashvili, ${ }^{1}$ Estrella Lasry, ${ }^{3}$ Sandra Wagner Bauer, ${ }^{1}$ \\ Carolina Jimenez ${ }^{3}$
}

To cite: Eibs T, Koscalova A, Nair M, et al. Qualitative study of antibiotic prescription patterns and associated drivers in Sudan, Guinea-Bissau, Central African Republic and Democratic Republic of Congo. BMJ Open 2020;10:e036530. doi:10.1136/ bmjopen-2019-036530

- Prepublication history for this paper is available online. To view these files, please visit the journal online (http://dx.doi. org/10.1136/bmjopen-2019036530).

Received 19 December 2019 Revised 17 August 2020 Accepted 18 August 2020

Check for updates

(c) Author(s) (or their employer(s)) 2020. Re-use permitted under CC BY-NC. No commercial re-use. See rights and permissions. Published by BMJ.

${ }^{1}$ Vienna Evaluation Unit, Medecins Sans Frontieres,

Vienna, Austria

${ }^{2}$ Infectious diseases departement, Slovak Medical University, Bratislava, Slovakia

${ }^{3}$ Medical departement,

Medecins Sans Frontieres, Barcelona, Spain

Correspondence to Dr Alena Koscalova; alena.koscalova@gmail.com

\section{ABSTRACT}

Objectives The objective of this study was to address the knowledge gap regarding antibiotic use in Medecins Sans Frontiéres (MSF) projects located in Africa by exploring antibiotic prescription and consumption habits and their drivers at different healthcare levels.

Design This study used an exploratory study design through thematic analysis of semistructured, in-depth interviews, focus group discussions (FGDs) and field observations in order to understand the main drivers influencing current antibiotics prescription habits and consumption habits of patients in different geographical settings.

Setting The study took place in MSF centres and towns across four countries: Guinea-Bissau, Central African Republic (CAR), Democratic Republic of Congo (DRC) and Sudan.

Participants 384 respondents participated in the study, which includes project staff, prescribers, community members, patients, among other groups.

Results Treatment protocols were physically present in all countries except DRC, but compliance to protocols varied across contexts. A failing health system and barriers to accessing healthcare were perceived as major drivers of overuse and inconsistent prescription practices. Patient demands influenced prescription decisions, and self-medication was commonly reported in the context of failing health systems. Additionally, there was a strong demand for quick cures and communities preferred injections over pills. Patients tended to stop antibiotic treatment once symptoms abated and had major gaps in understanding antibiotic intake instructions and functions.

Conclusions While there were specific findings in each context, the larger trend from these four MSF projects in Africa indicates widespread use of antibiotics based on unclear assumptions, which are often influenced by patient demands. There needs to be a broader focus on the balance between access and excess, especially in such fragile contexts where access to healthcare is a real challenge.
Strengths and limitations of this study

- The study successfully triangulates data between in-depth interviews and focus group discussions (FGDs) with 384 respondents (different groups of providers and patients) across four diverse countries.

- Participation in in-depth interviews and FGDs was more skewed toward men in general, but women's associations were specifically included in the FGDs to overcome this imbalance.

- The study did not include nomadic populations and local medicine vendors in Guinea-Bissau, but they were sampled in all other countries.

- Observation bias may have limited the validity of our findings, as health staff may have amended their behaviour or put more effort into explanations compared with usual practices.

\section{INTRODUCTION}

Antibiotics or antibacterial drugs are medications that kill or slow down the growth of bacteria. Although antibiotics have saved many lives during the last century, the development of resistance to commonly used antibiotics hinders their benefits in controlling infectious diseases. This is of serious concern, especially in developing countries where infectious diseases remain the main cause of morbidity and mortality, and access to newer antibiotics, which can be lifesaving when treating infections caused by resistant bacteria, remains poor. ${ }^{1}$

In 2015, Medecins Sans Frontiéres (MSF) recognised antibiotic resistance $(\mathrm{ABR})$ as a priority and called for the elaboration of a roadmap to tackle ABR in MSF projects. The MSF ABR Task Force was consolidated in 2017 with three main working areas: infection prevention and control, antibiotic 
Table 1 MSF presence and project context

\begin{tabular}{|c|c|c|c|}
\hline DRC & CAR & Sudan & Guinea-Bissau \\
\hline $\begin{array}{l}\text { Health services are provided } \\
\text { through } 30 \text { Ministry of Health } \\
\text { (MoH) health structures: } \\
21 \text { health centres, } 8 \text { health } \\
\text { posts and } 1 \text { referral hospital. } \\
\text { In the hospital, MSF } \\
\text { is neither involved in } \\
\text { consultations nor in the } \\
\text { prescription of antibiotics, } \\
\text { the role of MSF is restricted } \\
\text { to supervision and support. }\end{array}$ & $\begin{array}{l}\text { The MoH operates one } \\
\text { health centre in Kabo, } \\
\text { which has been managed in } \\
\text { partnership with MSF since } \\
2006 . \\
\text { It was reconstructed by MSF } \\
\text { and extended to a secondary } \\
\text { health facility providing } \\
\text { maternity, paediatrics, } \\
\text { internal medicine, surgery, } \\
\text { HIV/AIDS, mental health, } \\
\text { disease prevention, } \\
\text { Expanded Program on } \\
\text { Immunizations (EPI) and } \\
\text { vaccinations. }\end{array}$ & $\begin{array}{l}\text { Since 2007, MSF } \\
\text { provides health } \\
\text { services through its } \\
\text { centres in Tawila, } \\
\text { which have an in- } \\
\text { patient department } \\
\text { (IPD) and out-patient } \\
\text { department (OPD) } \\
\text { with two mobile } \\
\text { clinics in Um Jalpakh } \\
\text { and Tibido. } \\
\text { Close to nomadic } \\
\text { population migration } \\
\text { routes and settlement } \\
\text { points. }\end{array}$ & $\begin{array}{l}\text { Toward the end of } 2015, \text { MSF } \\
\text { launched a new project in the } \\
\text { main paediatric hospital of the } \\
\text { country (National Hospital of } \\
\text { Simao Mendes) in the capital } \\
\text { Bissau, which had a paediatric } \\
\text { intensive care unit. } \\
\text { The Simao Mendes Hospital } \\
\text { serves as a referral hospital for } \\
\text { the whole country and since } \\
\text { April } 2018 \text { serves as the only } \\
\text { MSF project in the country. }\end{array}$ \\
\hline
\end{tabular}

CAR, Central African Republic; DRC, Democratic Republic of Congo; MSF, Medecins Sans Frontiéres.

stewardship and diagnosis (access to bacterial culture and antibiotic susceptibility testing), and surveillance.

This study was commissioned to provide an insight into antibiotic use and its drivers in MSF projects. It aimed to address the knowledge gap regarding antibiotic use in MSF projects located in Africa by exploring antibiotic prescription and consumption habits and integrating insights from patients, communities and prescribers in project areas in four countries: Guinea-Bissau, Central African Republic (CAR), Democratic Republic of Congo (DRC) and Sudan.

The overall objective of the study was to obtain a thorough understanding of the use of antibiotics (by prescribers and by patients) and of the main drivers within different contexts in MSF interventions by using a mixed-methods approach. Specifically, the study

1. Explored the reasons for the current prescription habits and factors influencing health workers prescription per group of prescribers (doctors, nurses, other health workers).

2. Investigated patients' demands on and expectations of antibiotic prescription and explored explanatory factors.

3. Explored the consumption habits of patients and underlying reasons for consumption.

Table 1 summarises the project context and MSF presence across all four countries.

A compilation of internal MSF data shows high levels of resistance in burn sepsis and war wounded patients, particularly in the Middle East and among neonates, including some recent outbreaks of resistant pathogens in Haiti and CAR. A study in malnourished children in Niger demonstrates that around 94\% of children are colonised with extended-spectrum beta-lactamaseproducing Enterobacteriaceae (ESBL-E) at discharge. ${ }^{2}$ In South Kivu province of DRC, antibiotic-resistant isolates were commonly resistant to antimicrobials used to treat urinary tract infections: the ESBL-phenotype was found in approximately $93 \%$ of resistant isolates. ${ }^{3}$ In general, there are very few, if any, country-specific studies for Guinea-Bissau, Sudan and CAR. However, a multicountry study exploring the distribution of ESBL-associated Gram-negative bacteria found a sub-Saharan Africa genotypic ESBL prevalence of $9.3 \%$ and phenotypic ESBL prevalence of $12.1 \%$ based on data from nine countries, including Sudan and Guinea-Bissau. ${ }^{4}$ We were unable to find any studies that systematically explored the drivers or prescription behaviours related to antibiotic use, which might be able to explain high levels of ABR.

This study aims to provide an insight on antibiotic use and its drivers in MSF projects. It aims at filling the knowledge gap regarding the antibiotic use in MSF projects located in sub-Saharan Africa, exploring antibiotic prescription and consumption habits and their drivers at all the levels of healthcare (community, health centres (HCs) and hospital).

\section{METHODOLOGY}

A qualitative and exploratory nature of inquiry constituted the analytical framework for the study. Interviews, focus group discussions (FGDs), case discussions and field observations were conducted to understand the main drivers and factors influencing the current antibiotics prescription habits, as well as the different demands, expectations and consumption habits of patients in different geographical settings. All interviews and FGDs were guided by semistructured topic guides (see online supplemental file 1), which were developed prior to data collection and refined iteratively during the course of the evaluation.

In each project area (except Guinea-Bissau), the population involved in this evaluation comprised: 
a. Prescribers within the MSF projects described above (professions vary depending on the project setup; prescribers can be doctors, nurses or clinical officers).

b. Actual patients from MSF health facilities at the drug dispensary.

c. Community members in the project's catchment area and community health workers.

d. Drug sellers/shopkeepers/dispensers.

e. Health professionals and management in MSF projects and $\mathrm{MoH}$ staff.

Ethics Review Board (ERB) approval was obtained for all project sites, except Guinea-Bissau. Hence, the evaluation in Guinea-Bissau had to be confined to MSF project data and MSF staff as respondents, rather than community-based discussions.

Our study used a maximum variation purposive sample to examine a diverse range of cases that are all relevant to the research question. This allowed us to gain insight from many different angles. The selection criteria and procedures for each country have been detailed below.

\section{Sudan}

In Sudan, interviews and FGDs were conducted in Tawila town (with the original town residents), and in the internally displaced person (IDP) camps of Rwanda, Argo and Borgo, which represent the different waves of displacement to Tawila. Interviews and one FGD were also conducted in the Um Jalpakh area where the mobile clinic operates once a week and the population includes nomadic groups. The choice of participants for individual interviews in the community and the clinic was focused mainly on gender with an emphasis on women. FGD participants were approached through community leaders and the MSF community health worker (CHW) network. The study sites were the Tawila Health Centre and Um Jalpakh Mobile Clinic. In addition, the communities and private pharmacies in Tawila town, Rwanda, Argo, Borgo and Um Jalpakh area were also included.

\section{Guinea-Bissau}

In the Bissau project, interviews were conducted exclusively with MSF staff, namely, doctors, supervisors and coordinators. Selection criteria for the prescriberinterviewees included the following: hired on MSF contract and working in either the emergency room (ER), pediatric intensive care unit (PICU) or neonatal intensive care unit (NICU); and working in the project for at least
6 months. Participants were included using purposive sampling, with an added emphasis on representativeness of the sample by capturing a diversity of the prescribers, that is, varied seniority of the position (service supervisors vs prescriber doctors), length of work experience (from more than 2 years to just over 6 months) and gender (male and female). The difference in age was not an issue because all doctors belonged to a similar age group (between 25 and 35 years old).

\section{Democratic Republic of Congo}

In the Lulingu DRC project, MSF staff were not functioning as prescribers; instead, the supervising Ministry of Health $(\mathrm{MoH})$ (Bureau Centrale de la Zone de Santé de Lulingu or BCZ) staff were responsible for prescriptions. Hence, interviews were conducted with MSF supervisors as well as with $\mathrm{MoH}$ prescribers.

The population of the zone de santé is quite heterogeneous, comprising a mix of different ethnic groups, which have been merged together by migration and forced displacement. Therefore, in the context of Lulingu, the sites and interviewees were not selected according to criteria of ethnicity, but rather according to criteria such as the level of vulnerability and access to care (or remoteness from health facilities). FGD participants were approached via the chef de quartier of their residential area who was informed about the evaluation, the objectives, the process and the implications of the participation.

\section{Central African Republic}

Given the vast variety of ethnic groups living in and around Kabo, an effort was made to cover at least one respondent from each group. Additional factors which governed the choice of participants included distance to the $\mathrm{HC}$ and affiliation with French/Sango or Arabic speaking groups, respectively. FGD participants were approached by the chef de quartier of their residential area who was informed about the study, the objectives, the process and the implications of the participation.

Tables 2 and 3 present the total number of IDIs and FGDs conducted with participants across the four contexts.

In total, 112 respondents participated in IDIs and 272 in FGDs across all four countries.

Table 2 Number of individual, in-depth interviews

\begin{tabular}{|c|c|c|c|c|}
\hline & Sudan & CAR & DRC & $\begin{array}{l}\text { Guinea- } \\
\text { Bissau }\end{array}$ \\
\hline Prescribers & 12 & 3 & 5 & 6 \\
\hline Community members and patients & 24 & 16 & 22 & N/A \\
\hline Total & 38 & 24 & 40 & 7 \\
\hline
\end{tabular}

CAR, Central African Republic; CHW, community health worker; DRC, Democratic Republic of Congo. 
Table 3 Number of FGDs

\begin{tabular}{|c|c|c|c|c|}
\hline & Sudan & CAR & DRC & $\begin{array}{l}\text { Guinea- } \\
\text { Bissau }\end{array}$ \\
\hline Project staff and local authorities & 2 & 6 & 2 & 1 \\
\hline Total number of participants in FGDs & 148 & 81 & 41 & 2 \\
\hline
\end{tabular}

CAR, Central African Republic ; DRC, Democratic Republic of Congo; FGDs, focus group discussions.

\section{Data collection and analysis}

Study visits and data collection took place in all countries between 8 July 2018 and 27 November 2018 (including travel days). Qualitative data collection comprised of individual in-depth interviews, FGDs and observations in the health structures and exit interviews in health facilities. Data collected from the interviews and FGDs consisted of hand-written interview notes taken in situ during the conversations (assisted by a translator). Direct observation was conducted while sitting in on consultations at the OPD and observing drug dispensing behaviour in MSF and private pharmacies, as well as participating in MSF staff meetings, case discussions and informal discussions with MSF staff and community members.

The interviewers took detailed notes during the interview and transcribed each interview verbatim within 48 hours. Given the context of intensified security concerns in DRC and CAR, audio recordings were not considered due to concerns regarding trust and denial of participation. A face-to-face discussion without recording devices was considered as the more optimal approach. The researchers then used a Microsoft Word document to separate interview data into logical chapters and subchapters. Systematic manual coding (without double coding) ensured that all the content was coded, and the codes corresponded with main research themes. After sorting and structuring the data, researchers analysed the data using a systematic, iterative process of identifying and comparing common themes, but also considering divergent or dissenting perspectives. Computer-assisted qualitative data analysis software was not used as part of this process.

Data collection was triangulated through various interviews, field observations and FGDs to check credibility and clarify discrepancies across multiple perspectives. Each interview was cross-checked in following interviews, and the codes and themes emerging from each transcript were validated against other transcripts. If the same codes kept emerging, it was seen as a persistent pattern and coded as a theme. Data were triangulated between respondent groups and interviewers as well.

Observations were conducted by the interviewers, who were trained in social anthropology and participant observation. A detailed checklist was not used because the procedures and course of events in the HCs were unknown and impossible to anticipate prior to arrival. Given the exploratory nature of inquiry, observations focused on patient behaviour and understanding of procedures, as well as interactions with medical staff. Observed behaviours differed considerably from one setting to the next, and a checklist was deemed to hamper the process in comparison to detailed open coding and observation.

Qualitative data analysis and collection occurred concurrently using Mayring's thematic content analysis framework, and continued until thematic saturation was attained. ${ }^{5}$ Based on repeated data screening, codes and categories were identified and attached to the statements. Statements with the same categories were brought together and interpreted in relation to each other and structured along the research questions. Coding was performed manually and no software was used, primarily due to resource constraints. In the analysis, special attention has been given to presenting data along with its context and also presenting deviant cases. Each response corresponding to a certain topic from the interview guide was coded (assigned a certain colour) and then categorised with respect to its topic. In the analysis, we categorised patterns that recurred frequently as overarching themes. Less common themes were also included unless they represented singular opinions, and we included a separate category for responses that demonstrated an exceptional misunderstanding of antibiotic use.

\section{Ethical considerations}

All participants provided informed consent after an explanation of the study objectives and procedures. This was done in written form or orally in case written consent was not appropriate.

\section{Patient and public involvement}

Patients and/or the public were not involved in the design, conduct, reporting, or dissemination plans of this research.

\section{RESULTS}

The results have been presented as major thematic areas in two domains: underlying factors of antibiotic use from the perspective of prescribers and consumption habits from a community perspective. 
Underlying factors of antibiotic use (prescriber perspective) Treatment protocols are physically present in all countries except $\mathrm{DRC}$, but compliance to protocols varies

Treatment protocols were widely used by prescribers in Sudan. The protocols were seen on every desk and on some of the phones (all those with smartphones) of the prescribers, and were mainly used by prescribers for complicated cases and as study material.

Prescribers of all three departments (ER, PICU and NICU) in the Bissau project were generally aware of treatment protocols and there was a common perception of compliance to protocols among supervisors:

The doctors prescribe antibiotics very correctly, according to the protocols. 90\%-95\% are prescribing correctly. The only question is the diagnosis - how well the differential diagnosis is made. (MSF Supervisor, Guinea Bissau)

Most doctors in Guinea-Bissau reported that they knew the guidelines and dosages by heart already, so they consulted the protocols only in case of rare conditions. However, language was noted as a potential barrier to the systematic use of protocols in Sudan. Although it was said that some protocols were available in Arabic, only the English copies were seen by the evaluation team. Additionally, while treatment protocols were known to be present in Tawila, Sudan, they were usually not available for routine work by staff and there were contradictions between national and MSF guidelines.

Data from interviews with prescribers, OPD patients, community members and field observations at the HC dispensary suggested that MSF staff in Kabo, CAR complied with the treatment protocols with regard to antibiotic prescriptions. Prescribers at all levels (supervisors and national staff) had sound knowledge of and were aware of the risks of overprescription and ABR.

That being said, however, DRC was a notable exception among all countries because protocols were not present in all facilities. In addition to the lack of copies, compliance and use of the protocols was questionable in DRC. Staff complied with the protocols predominantly in $\mathrm{HC}$ Tchonka, but compliance was weakest in the paediatric ward of the Lulingu Hospital. MSF supervisors mentioned some practical issues which may explain inconsistent compliance to the protocols:

Health staff often have problems to identify the proper dosage ... although there are protocols and guidelines pinned to the wall ... either they forget to check, they do not care, they do not want to accept [treatment] protocols, or they do not understand how to calculate dosages, for example, $\mathrm{mg} / \mathrm{kg}$ for children. (MSF supervisor, DRC)

\section{Failing health systems are perceived as major drivers of overuse} among prescribers

Given the unregulated and uncontrolled dispensation of antibiotics at local pharmacies, prescribers and supervisors in Guinea-Bissau assumed a widespread presence of ABR. This was believed to be linked with the underdeveloped and understaffed primary and secondary healthcare system, which prescribes antibiotics widely, lacks medical supplies, and requests payment from patients to access medical care.

It may be that we have higher resistance against antibiotics than assumed by the protocols, because in the primary health care they use amoxiclav and ceftriaxone a lot...many times, they don't respond to the ATB that we have in our protocols. We then re-check the diagnosis and go for $2^{\text {nd }}$ or $3^{\text {rd }}$ line, but we do not know about the resistance patterns. (MSF supervisor, Guinea-Bissau)

Many interviewees mentioned that some drugs, such as amoxicillin or Amoxicillin-Clavulanate, were compromised due to their widespread prescriptions in the primary health centre (PHC) and uncontrolled use in the communities. Unpaid doctors were largely absent in the PHC and nurses were left to make diagnosis and prescription decisions; often, this contributed to overprescription of antibiotics.

According to supervisors and managers, prescribers followed up closely according to the protocols, but diagnostic accuracy and differential diagnoses were problematic. This was mainly due to limited availability of some laboratory tests. As one prescriber put it:

We are based on the clinical signs. In case if the diagnosis does not work, we don't have enough laboratory tests such as electrolytes check; echocardiography needs referrals and approval. (MSF prescriber, Guinea-Bissau)

Similarly, in Sudan, a large number of patients came to the OPD, so prescribers and dispensers had limited time with each patient. On average, the OPD medical staff spent approximately 5 min per patient, although the MSF guidelines stipulate that OPD consultations should take around 20 min per patient. In the IPD, the situation was better due to the smaller number of patients.

In the OPD as well as in IPD in Sudan, several register books were filled in parallel, as each prescriber was assigned his/her own register book. Hence, information on previous consultations and treatment could not be easily traced if the patient was other medical staff. This was especially problematic, given the fact that most patients did not know the name of the drugs they were prescribed; as a result, oral history taking would not suffice as a replacement.

Additionally, in Sudan, most patients were seen by medical assistants. Doctors were working mainly in the ER/IPD. Medical assistants mentioned that they felt insecure at times with regards to correct prescriptions.

I use MSF guidelines but sometimes they do not suffice so I need a doctor's help. (Medical Assistant, Sudan) 
In CAR, high workloads, especially at the OPD, resulted in short consultation times and inadequate anamnesis. Prescribers complained that they lacked time and often felt pressure related to the high workload.

Four consultants do 200 patients a day ... they cannot give a good and thorough consultation ... and there is no time for a proper explanation. (MSF health educator, CAR)

During the observations of consultations in the paediatric and adult OPD in CAR, prescribers reviewed previous treatments, but patients were not asked about a potential allergy to antibiotics. Prescribers did not properly explain antibiotic intake.

\section{Overprescribing antibiotics as a precautionary measure}

In some instances, prescribers in Sudan claimed to consciously prescribe either more antibiotics than recommended, or prescribe antibiotics based on certain assumptions. For instance, due to the limited number of follow-up visits (usually only done if the patient was in a bad condition), prescribers found it difficult to adopt a wait-and-see approach. When in doubt, prescribers opted to prescribe antibiotics immediately because they claimed that a follow-up visit, even after assuming proper compliance by patients, might represent a burden for the patient and family in terms of cost, distance, or time.

Some prescribers in Tawila explained that they preferred to err on the side of overprescription rather than underprescription due to the generally low levels of hygiene and nutrition in the area, and hence the possibility of other diseases being present.

The general standard of hygiene both personal and environmental is substandard hence there are a lot of infections and need to use antibiotics. (MSF prescriber, Sudan)

Similarly, in DRC and Bissau, it was observed that medical doctors prescribed more antibiotics than necessary to be 'on the safe side'. Nurses at the PHCs in Bissau prescribed antibiotics based on their observations of doctors' prescription habits. If they saw a doctor prescribing an antibiotic to a sick person with a cough and a fever, they would do the same, without knowing that the two cases may be due to different diseases. Furthermore, doctors in the PHCs prescribed without any protocols and were prone to heeding demands from patients, which reportedly contributed to high prescription rates.

Additionally, in DRC, the necessity to use all medications in the stock before they expired seemed to drive prescription practices:

When the expiry date is near, I tell the doctor that he should prescribe more ... it is better to give the antibiotics to the patients instead of throwing them away. (Hospital pharmacist, DRC)
Patient demands for antibiotics influenced prescribers in different ways across countries

Prescribers at all levels (doctors and nurses) reported a strong demand for antibiotics and injectable drugs from the population. According to the medical director, $99 \%$ of patients typically asked for antibiotic treatment.

Yes, it is often to demand drugs. Sometimes they come without a sick person and request a certain drug. They want antibiotics. (MSF prescriber, Guinea-Bissau)

All prescribers who were interviewed in Guinea-Bissau stated that patient demands or their ethnicity and age group did not influence prescription behaviour, but this information was not triangulated through other methods. However, in Sudan, prescribers reported feeling obliged to give medication in order to build confidence with patients, especially when patients travelled from afar to access health services and had very limited access to 'modern' medicine facilities. This behaviour was supposed to maintain patient trust in the prescribers' knowledge and abilities, as the general societal perception was that proper treatment required a prescription. Prescribers claimed that, in such cases, they mostly prescribed painkillers (paracetamol/diclofenac) or a vitamin supplement, but not antibiotics.

According to prescribers in CAR, marginalised groups (Mbarara, Lithos) or members of the Muslim communities in Kabo (Salamat, Chadian) requested 'powerful' medications the most compared with other groups. In the major dispensaries across the CAR project, there were two distinct types of drug sellers: those who were entitled to sell medications without a prescription and those who were not. Many of these sellers even practised home visits. However, interviews with the drug sellers revealed a low level of education (6 months with diploma or interrupted education). These drug sellers adapted to the demand and consumption habits by providing 'powerful' medicine (antibiotics and injectable malaria treatments) and by giving injections.

Amoxi is the most used antibiotic in families ... they get it from the PDS ... on the markets (pharmacies) they mostly do injections ... the most given injections are ceftriaxone, penicillin and gentamicin. (MSF health educator, CAR)

Finally, in DRC, prescribers perceived that many community members sought medications according to their previous experiences to avoid costs, lengthy admittance procedures and inefficient diagnoses in the health facilities.

Many come and ask for a certain medication without knowing what disease they have...they (the clients) say: now I came all the way and you only give me paracetamol? They all ask for antibiotics. (Local drug seller, DRC)

All pharmacies who were visited in DRC admitted handing out antibiotics without a prescription. Only 
one out of three pharmacists who were interviewed had finished his education (as a trained nurse).

Patients want the product (antibiotics) that they have in mind ... they do not want a counselling ... they say they just come to buy not to get advice. (Local drug seller, DRC)

\section{Barriers to accessing free quality health care}

Access to healthcare in Sudan peripheries was deemed to be challenging. In the catchment area, no other provider was offering free healthcare services, which prompted an increased reliance on self-medication from local pharmacies.

If MSF leaves us, we will die because we cannot afford to pay for treatment. (Community member, male, Sudan)

Although some areas were partially served by the mobile clinics, many patients had to travel long distances to access medical care. In addition, even the mobile clinics were cut-off during the rainy season, forcing the population to trek to Tawila on their own to access health service.

Time constraints were another barrier to access MSF health services. Waiting times on busy days could take up to 5 hours because of the huge number of patients and the comparatively limited number of prescribers. Especially during the periods when people were busy with farming, patients tended to avoid going to the $\mathrm{HC}$ and either purchased medication from local pharmacies or used medication previously prescribed to them by MSF. Time constraints were also found to be a nagging issue among community members in CAR. The mobile populations (cattle herders) in CAR found it difficult to leave their animals alone for a whole day to seek healthcare. They preferred to visit the pharmacy to receive an injection, which was expected to immediately enable them to return to their herds.

Moreover, the admission procedures in the Kabo project in CAR were particularly problematic and presented a considerable barrier to accessing healthcare. All communities in and around Kabo perceived admission procedures at the $\mathrm{HC}$ as unsatisfactory, unfriendly, hostile or discouraging. The complaints mainly referred to the system of triage. Emergencies were treated with priority and patients without fever had to wait the longest and risked being sent home without being seen.

They tell us to come again tomorrow ... but the problem is NOW ... we don't come to the HC for no reason. (Community member, CAR)

Furthermore, perceived discrimination functioned as an additional barrier to seeking care in CAR. Most prescribers (MSF national staff) were Christians from Bangui or urban centres. Some groups of patients felt uncomfortable spending a lot of time among members of the other groups. In interviews, some respondents from nomadic groups (Mbarara), remote indigenous populations (Lithos) and resident Muslim populations counted frustrating experiences in the HCs and attributed these to discrimination.

Last time I came they did not admit me ... they discriminate me because I am Mbarara ... they didn't even take my temperature and they sent me home ... after travelling for $20 \mathrm{~km}$ I bought medication at the market ... but I would rather get treatment from MSF ... this time they treated me. (Patient, CAR)

Delays in the waiting area were often interpreted as discrimination based on ethnic reasons. Women from the ethnic majority (Sara, Banda) and from the local women's association reported unfriendly admission procedures, which occasionally supported their decision to avoid the HC and look for treatment in the local pharmacies. Poverty and insecurity were reported to be another common reason for avoiding admission and consultation fees in HCs in DRC.

People know that it is risky to go to the "infirmière pirate" (pirate nurses) but they do it out of poverty and because they do not give good treatment in the hospital. (CHW, DRC)

At night, especially when there is insecurity, we go to the pharmacy in the neighborhood instead of the HC. (Community member, male, DRC)

\section{Consumption habits and underlying reasons (community perspective)}

Traditional medicine was usually used when MSF services failed Reverting to traditional/religious medicine was mainly found among patients with chronic illnesses or mental health issues in Sudan. The scope of traditional treatments used was mainly limited to the use of herbs and/or Mihaya (traditionally water blessed with incantations and readings from the Quran).

These are old people treatments. Nowadays we go to the doctor... most of our old people who knew about these things are dead anyway ... we still use Mihaya and similar things for protection of our children. (Community member, female, Sudan)

In CAR, even though MSF prescribers reported frequent cases of patients who consulted traditional healers or relied on homemade remedies before coming to the HC, most community respondents claimed that MSF was seen as the first choice in health-seeking behaviour, with the pharmacy as second choice, and the traditional practitioner only as third or last choice.

When MSF treatment is not effective or when the same treatment is given again people go to see the healer ... but most of us go to the HC ... people know that the quality is higher. (FGD youth association to combat HIV, CAR) 
In DRC, the majority perceived modern medicine as the first choice of treatment, with the exception of singular cases among very precarious and marginalised populations. A common practice included applying a mix of antibiotics to open wounds to accelerate the healing process (ie, crushed chloramphenicol mixed with ampicillin). Such concoctions were also offered in local pharmacies.

In Guinea-Bissau, however, traditional medicine was still the preferred first choice. Respondents perceived traditional healers to be effective for some conditions, but not for others.

\section{High rate of self-medication in communities and explanatory} factors

Easy access to antibiotics without prescriptions in local pharmacies exacerbated the problem of self-medication, and many sellers were not trained pharmacists. If the medication was not available at the pharmacy, the drug seller occasionally sold another drug which 'had the same effect'.

For instance, in Sudan, all medications were available without prescription in local shops. These local pharmacies were run by persons with some level of medical training (nurses/medical assistants) and operated under an $\mathrm{MoH}$ license as drug stores. The drugs they provided were purchased from certified drug company agents in Elfashir. Sometimes, customers asked for medicines not by mentioning their categories or names, but either by showing a pill or by describing shape and colour. During an interview with a local shopkeeper, one customer approached him with a pill in an old plastic bag and asked if the same was available for purchase.

Prescribers in CAR suspected that marginalised populations (Mbarara, Lithos) regularly resorted to self-medication (including injectable antibiotics). Community-based discussions from CAR revealed that patients stocked their prescriptions at home (as a reserve) instead of taking the whole treatment because they wanted to avoid the procedures at the admission/ triage during their child's illness.

As for DRC, certain drugs were not available in the hospital/HCs, but they could be found on the private market. These medications were also cheaper than the ones sold in the hospital dispensary. Thus, if a prescriber lacked medication or refused to prescribe an antibiotic, the patient could easily obtain the treatment from a local provider.

\section{High demand for quick relief cures meant that all forms of} treatment were not equally accepted by communities

Self-medication may also be driven by a desire for quick relief and easy access to drugs (including antibiotics) in local pharmacies, particularly in CAR and DRC. Respondents stated that they sought products that were perceived as effective and provided quick relief, particularly injectable drugs (among the antibiotics) in local pharmacies.

Community members in DRC often expressed their frustration about children getting sick again only a few weeks after a visit to the health services and being prescribed the same 'ineffective' medication again and again.

In the hospital they release patients too early; the disease keeps coming back ... when you go to the hospital again the nurse says, 'now you come again' and prescribes the same medication. (community member, female, DRC)

In general, patients preferred intravenous solutions, as stated by community members and healthcare staff. solutions were considered as the most potent form of medication. Injections were slightly less effective, followed by syrups. Pills were perceived as the least potent treatment form. Most prescribers shared historic anecdotes of seeing pills being thrown out by patients near the facility. This was very much the case in Sudan, although there was a reported change in behaviour as the exposure of communities to modern medicine and community health education increased in Tawila.

In addition, intake of pills was often perceived as unpleasant or difficult with regard to taste. It also required sufficient food and clean water. Side effects caused by pills consumption (vomiting, stomach problems, nausea, drowsiness) were repeatedly mentioned as a reason to stop/interrupt treatment.

I am satisfied with pills instead of injections ... but here, especially children and elder people do not tolerate pills ... they spit or they stop because of side effects and because they don't have enough food.

(Community member, female, CAR)

Treatment with pills was relatively new in the CAR region and associated with the opening of the MSF project; administration of injectable drugs (mostly artemether and quinine but also ATB) was the most common way of treatment. Hence, a considerable part of the population-especially those who were not reached by MSF health educators-still preferred powerful, injectable drugs and rejected oral treatment.

All interviewees (community members, patients, CHWs and prescribers) in DRC confirmed the preference for injectable medications over oral intake. Reasons given for this preference ranged from faster effect, stronger efficiency, better tolerance to psychological effects (infusion means hope and comfort) and feelings of getting 'a real treatment' (as compared with the superficial services usually offered in the hospital).

Injectable drugs are more effective ... they have immediate effect. (Community member, female, DRC)

Community members associated treatment for some diseases with antibiotic use

Some communities, especially in CAR, clearly associated particular medical conditions such as RTIs with amoxicillin treatment. This was found to be the case among a majority of Kabo residents (not so much for the mobile populations around Kabo). In particular, malaria 
symptoms such as stomach problems, were expected to be treated with antibiotics.

Similarly, in DRC, malaria was often associated with antibiotic prescriptions. Community members stated that they usually took ACT together with antibiotics, assuming that this is the standard treatment. Furthermore, reports indicated that women who underwent caesarean sections found it necessary to receive antibiotics to cure the wound and requested antibiotics for deliveries. Since they were released soon after birth (2 days), they extended the antibiotic treatment they received in the hospital with additional products purchased in local pharmacies.

Women are unsatisfied when they only get paracetamol after a caesarean ... when they have pains, they buy antibiotics in the pharmacy ... they ask for what they remember from their last delivery and buy amoxi, gentamicin and Peni V (penicillin). (CHW, DRC)

A repeatedly stated conclusion from community respondents (mothers as well as drug sellers) was that cough, predominantly in children, must be treated with amoxicillin.

I give amoxi to my child when it coughs ... when the treatment is over, and it is still coughing I go and buy more amoxi" (Community member, female, DRC).

\section{Incomplete intake of antibiotic treatment and explanatory factors}

Patients in CAR indicated that they tended to stop antibiotic treatment after symptoms abated:

There are many women who do not abide to treatment recommendations ... they keep parts of the treatment for later when the child gets sick again ... or they give it to other children in the family ... all that because they want to avoid going through admission in the HC. (Community member, female, CAR)

Similarly, in Sudan, even if patients were given the full course of treatment, some patients stopped the intake when they felt better and then either kept medications for later use or used them as shopping guidance to show to local drug sellers and obtain similar medications in the future.

Some patients, especially mothers, shared their medications with children with similar symptoms.

I always keep some medication in the house. You never know when you will need it and sometimes I can't get to the clinic, so I keep some of the drugs they prescribe me to use until I can find time to go to the clinic. Sometimes I borrow from my neighbours. (Community member, female, Sudan)

Incomplete intake of antibiotic treatment was also linked to financial factors in other countries. For example, incomplete antibiotic treatment was commonly sold at the local pharmacies in CAR. Most patients in DRC reported that they could not afford to pay for the complete treatment and therefore had to buy antibiotics according to their budget (2-4 pills; only part of the whole prescription). They did not receive the full dose and therefore stopped the treatment before it was completed.

Similarly, when the MSF pharmacy was out of stock of a prescribed medication, some patients in Sudan secured them from the local pharmacies on the market. The majority tended not to buy the full course of medication prescribed because of cost and limited funds. This was particularly true for IDPs who had a very small income that hardly covered the cost of food.

I have to work all day to be able to buy a bowl of sorghum for my family. It costs 35 pounds... if I have to buy medicine for 60 , then they will go without food for two days. (Community member, IDP, female, Sudan)

In DRC, the medical system and the private sector supported incomplete treatment by giving out antibiotics in small doses (this was true at the hospital as well as HCs and private clinics/pharmacies). Due to the limited financial capacities of patients and relatively high costs of medications, prescribers tended to only hand out the amount of pills that patients could afford (eg, 4 pills of amoxicillin) instead of the full treatment (eg, 14 pills for 7 days). Patients were told to buy the rest when they had finished the first part or as soon as they could afford to buy more. Frequent shortages of medications in the $\mathrm{MoH}$ stocks also contributed to that practice.

There is a problem with some of the $\mathrm{MoH}$ prescribers: they prescribe the full treatment, but they only give half of the pills to the patients; the rest they sell in the community. (MSF supervisor, DRC)

Another major reason for incomplete intake of antibiotic treatment was that many patients did not understand the prescriptions which were provided. Most interviewees in CAR claimed to be satisfied with the given explanation by prescribers, but many could not reproduce the message or gave wrong answers with regard to their treatment. In Sudan, most community members described disinterest or even fear of knowing how drugs worked. Some prescribers expressed limited confidence in the ability of patients with limited education to grasp detailed explanations. At the hospital level in DRC, instructions were provided in the paediatric ward, where the atmosphere was noisy and distracting. When questioned afterward, most of the patients did not know what kind of medication was prescribed (ATB or other), whereas others did not understand the pathology.

Besides financial barriers and inadequate information retention, security was also mentioned as a hindering factor for complete intake in DRC:

I know I should take amoxi for seven days ... but I had to leave my house in Swiza because of the rebels ... I left my pills and my patient book when we had to flee 
... so I stopped treatment after 5 days. (Community member, female, DRC)

Additionally, language barriers represented a considerable challenge in CAR, especially for Arabic-speaking groups inside the OPD and at the dispensary. Many illiterate patients could neither relate to the symbols on the sachet (sun=morning/ moon=night, and so on) nor to the lines indicating the number of pills that had to be taken.

\section{Trust in prescribers' judgement and treatment plan}

Most respondents expressed a high level of trust in the judgement and prescription decisions of MSF health staff. Many respondents stated that this trust led to stronger compliance:

If you don't follow the doctor's orders, you don't get well. (Community member, female, Sudan)

According to HC staff, people who never went to school or who only visited school for a limited amount of time tended to follow doctors' orders without doubts due to high trust in modern medicine. Others, with higher education, tended to question the doctor's decision and occasionally requested different medications than the ones which were prescribed.

Community members in CAR continuously complained that symptoms kept coming back. Interview respondents claimed that prescribers did not change the treatment even if a patient returned several times with the same disease, thereby conveying the impression that prescribers did not question the effectiveness of a treatment.

The consultation is only between 3 and 5 minutes ... they don't check the carnet and they don't really listen when we tell them about our (health) problems ... there are people here who don't go to the HC because they know they will get the same treatment for a problem that doesn't go away. (Community member, CAR)

In general, all population groups in CAR perceived MSF as the best healthcare provider (highest quality, most professional and most affordable). However, effectiveness of treatment was questioned all too often, especially in the OPD. Many respondents described receiving medications that had become inefficient and powerless.

They always give the same treatment, we take it but we come back with the same disease ... they give us the same treatment again and so on ... that's the treatment we get at the HC. (Community member, CAR)

\section{DISCUSSION}

While there were specific findings in each context, the larger trend from these four MSF projects in Africa indicates a high level of antibiotic prescription based on unclear assumptions and often influenced by patient demands. Despite the clear presence of protocols in three out of four countries, compliance with protocols is inconsistent and requires further intervention. In addition, an inadequate or failing public health system presents major barriers to accessing care for patients in these countries, many of which have been ravaged by war or conflict for several decades. Many patients frequently rely on overthe-counter antibiotics and self-medicate without taking precautions to complete the full course of treatment. There is a high demand for consultations and a concomitant lack of time for prescribers to deeply examine each case and to provide proper explanation of the treatment and intake. Furthermore, beneficiaries live in extreme poverty and lack the time to travel to health facilities, which compromises proper care and follow-up services. This might explain the hesitance of both prescribers and patients to adopt the 'wait and see' approach that has proved useful in contexts where watchful waiting and observation are feasible. ${ }^{6-9}$

Previous studies from low-and-middle-income countries (LMICs) have reported that patient demands often play a salient role in inconsistent prescription practices. ${ }^{10-15}$ For example, Liu et al noted that rational prescribing practices are prone to weak control over prescribing behaviours, and $40 \%$ of prescriptions in a primary care setting in Hubei, China, contained an antibiotic, with $10 \%$ containing two or more antibiotics. ${ }^{14}$ Where antibiotic stewardship efforts have worked, they have relied on dedicated committees overseeing antibiotic use, prompt laboratory investigations, established guidelines for empiric prescribing and an easily accessible antibiogram. ${ }^{16}$ However, none of these components of an antibiotic stewardship strategy were in place in the four countries included in this study. Hence, our results serve as baseline data for MSF prior to the rollout of the ABR strategy in similar settings.

In the African context, studies have reported several inadequacies in the availability and implementation of antibiotic stewardship programmes. ${ }^{16-23}$ Fadare et al reported that even tertiary hospitals in Nigeria, one of the African countries where ABR plans have progressed most, lacked institutional capacity in that only $35 \%$ had a formal organisational structure and facility-based treatment recommendations based on local antimicrobial resistance patterns were only available in $24 \%$ of the facilities. ${ }^{16}$ A systematic review of ABR in Africa found that data on antimicrobial resistance was not available for $42.6 \%$ of African countries and the level of resistance to commonly prescribed antibiotics is significant. ${ }^{20}$ The challenges for ABR responses and plans in sub-Saharan Africa are huge and progress is difficult without the commitment of both governmental and nongovernmental health actors.

MSF is working to improve antibiotic prescription at all healthcare levels through antibiotic stewardship programmes, infection prevention and control, and access to microbiology and testing where feasible. MSF has already designed some context-adapted ABR responses in 
countries across the Middle East and similar approaches need to be developed for MSF projects in Africa. ${ }^{21}$

First, MSF must invest in understanding the contextspecific ABR profiles (ie, with regular studies at sentinel sites) and adapt treatment protocols accordingly. Protocols must be made available in local languages for each specific context. They also must be adapted and tailored to include medical conditions (ie, asphyxia in neonatal units or nosocomial infections) specific to each context in order to aid in differential diagnosis and management.

Second, since prescribers are vulnerable to patient demands, a larger effort must be made in health promotion to empower community members on the proper uses of antibiotics and support prescribers to develop their communication skills to achieve optimal adherence. Triage mechanisms at hospitals with a high OPD load seem to be poorly understood by the patient population, which jeopardises trust in prescribers. This can be particularly problematic in the long run, especially in countries like DRC which have commonly reported attacks on health workers in the context of an ongoing Ebola epidemic. ${ }^{23}$ Thus, health promotion has been identified as one of the key areas to develop within the ABR strategy and specific interventions will be implemented accordingly across the different MSF projects.

On the prescriber front, increased availability of pointof-care tests and algorithms might contribute to more accurate diagnoses of bacterial infections and patient history should be tracked more comprehensively. For instance, tools like MSFeCARE (an electronic clinical decision support system designed to guide primary healthcare workers to reach more accurate diagnoses and adapted prescriptions in the consultation process). The tool's intended use is for childhood illness under 5 years, but it could potentially be redesigned for other age groups, settings and medical conditions. Innovative options such as electronic medical records could be very valuable to track patient history, detect iterative consultations, follow drug history and diagnose nosocomial infections more accurately.

More broadly, reliance on self-medication, a preference for injections over pills and consumption of antibiotics without prescriptions are symptoms of an ailing health system that does not adequately meet the needs of patients. This is largely true in all four countries. In order to effectively address these practices, both access-related problems and issues related to drug regulations need to be tackled. Some interventions must be led at the $\mathrm{MoH}$ level with the development and subsequent implementation of National Action Plans on AMR that address the problem from a multisectoral, nationwide perspective. All four countries examined in this study are at an early stage in the implementation of national action plans. ${ }^{24}$ For instance, creation and implementation of national guidelines and a better controlled pharmacy sector are crucial for curbing the inappropriate use of antibiotics on national level. To reduce antibiotic demand and improve antibiotic consumption in the wider community, corresponding messages addressing their responsibility toward communities could be designed and communicated through adequate channels, such as mass media or community workers.

MSF is also including qualitative research as part of a broader advocacy strategy focusing on the balance between access and excess, especially in such fragile contexts where access to healthcare is a real challenge. In such countries, lack of access to life-saving antibiotics is as crucial as ABR, if not more pressing: one study in a Lancet series found that universal provision of antibiotics could prevent 445000 out of 590000 deaths from pneumonia in 101 countries. ${ }^{25}$ The focus moving forward must be both on improving access and reducing excess to incentivise better use of antibiotics, while working with patients, communities and prescribers through integrated health promotion strategies in stewardship programmes.

\section{Limitations}

Beyond geographic and time-related limitations, the sample in Guinea-Bissau did not include nomadic populations or local medicine vendors, but these groups were included in the remaining countries. Moreover, in CAR, the gender balance in some of the discussion groups was skewed as an open FGD format predominantly drew male participants. Women rarely joined in at public meetings and were often kept away in the case of nomadic groups and Muslim neighbourhoods. However, FGDs were held with women's associations to overcome this imbalance.

Observation bias could have affected the validity of our findings, as health staff may have amended their behaviour or put more effort into explanations compared with usual practices. Additionally, it is possible that respondents may not have felt comfortable sharing honest opinions in the presence of service providers. In some cases, community members were worried that MSF would exit the area and that affected some of the discussions. Furthermore, since an interpreter was required in some cases, there is always the added risk of inaccuracies in translation despite training measures in place.

There was also limited data from the community in Guinea-Bissau. During data analysis, double coding was not independently conducted by the interviewers, but codes were cross-checked between the team.

Since the study was qualitative and exploratory in nature, the findings cannot be generalised, but rather serve to illustrate important drivers among our patients. We aimed to mitigate this by including broad geographies, diverse respondent groups and a larger subset of respondents, although with purposive sampling.

\section{CONCLUSION}

Antibiotic prescriptions and consumption are driven largely by failing health systems and patient demands in CAR, DRC, Guinea-Bissau and Sudan, although the nuances in each context are different. Policy makers must 
balance the issue of excessive antibiotic use with access to care in fragile, conflict-ridden contexts.

Acknowledgements We thank the community members, healthcare staff, patients and their caretakers for their participation in the study. We also thank MSF field, coordination and headquarter teams for their support during the field research. We express our gratitude to the Ministry of Health in DRC, CAR, Guinea-Bissau and Sudan for facilitation of the evaluation.

Contributors TE, AK, MN and SWB wrote the manuscript. AK, TE, CJ and EL designed the research. PG, RGB, GK, MT, TE and AK performed the research and analysed the data.

Funding The authors have not declared a specific grant for this research from any funding agency in the public, commercial or not-for-profit sectors.

Competing interests None declared.

Patient consent for publication Not required.

Ethics approval Ethics approval was obtained from the Medecins Sans Frontieres Ethics Review Board (ID 1823).

Provenance and peer review Not commissioned; externally peer reviewed.

Data availability statement Data are available upon reasonable request. The data that support the findings of this study are available from Alena Koscalova, Director of Vienna Evaluation Unit, but restrictions apply to the availability of these data, which are under license for the current study, and so are not publicly available. Data are however available from the authors upon reasonable request and with permission of Alena Koscalova.

Open access This is an open access article distributed in accordance with the Creative Commons Attribution Non Commercial (CC BY-NC 4.0) license, which permits others to distribute, remix, adapt, build upon this work non-commercially, and license their derivative works on different terms, provided the original work is properly cited, appropriate credit is given, any changes made indicated, and the use is non-commercial. See: http://creativecommons.org/licenses/by-nc/4.0/.

\section{ORCID iD}

Alena Koscalova http://orcid.org/0000-0002-6838-0842

\section{REFERENCES}

1 Okeke IN, Laxminarayan R, Bhutta ZA, et al. Antimicrobial resistance in developing countries. Part I: recent trends and current status. Lancet Infect Dis 2005:5:481-93.

2 Woerther P-L, Angebault C, Jacquier $\mathrm{H}$, et al. Massive increase, spread, and exchange of extended spectrum $\beta$-lactamaseencoding genes among intestinal Enterobacteriaceae in hospitalized children with severe acute malnutrition in niger. Clin Infect Dis 2011;53:677-85.

3 Irenge LM, Kabego L, Vandenberg O, et al. Antimicrobial resistance in urinary isolates from inpatients and outpatients at a tertiary care hospital in South-Kivu Province (Democratic Republic of Congo). BMC Res Notes 2014;7:374.

4 Toy T, Pak GD, Duc TP, et al. Multicountry distribution and characterization of extended-spectrum $\beta$-Lactamase-associated gram-negative bacteria from bloodstream infections in sub-Saharan Africa. Clin Infect Dis 2019;69:S449-58.

5 Mayring P. Qualitative Inhaltsanalyse: Grundlagen und Techniken. Weinheim, Basel: Beltz Verlag, 2010: P.135.
6 Chao JH, Kunkov S, Reyes LB, et al. Comparison of two approaches to observation therapy for acute otitis media in the emergency department. Pediatrics 2008;121:e1352-6.

7 de la Poza Abad M, Mas Dalmau G, Moreno Bakedano M, et al. Prescription strategies in acute uncomplicated respiratory infections: a randomized clinical trial. JAMA Intern Med 2016;176:21-9.

8 Spiro DM, Tay K-Y, Arnold DH, et al. Wait-and-see prescription for the treatment of acute otitis media: a randomized controlled trial. JAMA 2006;296:1235-41.

9 Little P, Moore M, Kelly J, et al. Delayed antibiotic prescribing strategies for respiratory tract infections in primary care: pragmatic factorial, randomised controlled trial. BMJ 2014;348:g1606.

10 Nair M, Tripathi S, Mazumdar S, et al. Knowledge, attitudes, and practices related to antibiotic use in Paschim Bardhaman district: a survey of healthcare providers in West Bengal, India. PLoS One 2019;14:e0217818 https://doi.org/

11 Nair M, Tripathi S, Mazumdar S, et al. "Without antibiotics, I cannot treat": A qualitative study of antibiotic use in Paschim Bardhaman district of West Bengal, India. PLoS One 2019;14:e0219002 https:// doi.org/

12 Al-Homaidan HT, Barrimah IE. Physicians' knowledge, expectations, and practice regarding antibiotic use in primary health care. Int $J$ Health Sci 2018;12:18.

13 Davis M, Liu T-L, Taylor Y, et al. Exploring patient awareness and perceptions of the appropriate use of antibiotics: a mixed-methods study. Antibiotics 2017;6:23.

14 Liu C, Liu C, Wang D, et al. Determinants of antibiotic prescribing behaviors of primary care physicians in Hubei of China: a structura equation model based on the theory of planned behavior. Antimicrob Resist Infect Control 2019;8:23.

15 Baubie K, Shaughnessy C, Kostiuk L, et al. Evaluating antibiotic stewardship in a tertiary care hospital in Kerala, India: a qualitative interview study. BMJ Open 2019;9:e026193.

16 Fadare JO, Ogunleye O, Iliyasu G, et al. Status of antimicrobial stewardship programmes in Nigerian tertiary healthcare facilities: findings and implications. J Glob Antimicrob Resist 2019;17:132-6.

17 Antimicrobial stewardship in South Africa. react. Available: https:// www.reactgroup.org/toolbox/rational-use/examples-from-the-field/ antimicrobial-stewardship-in-south-africa/

18 Brink AJ, Messina AP, Feldman C, et al. Antimicrobial stewardship across 47 South African hospitals: an implementation study. Lancet Infect Dis 2016;16:1017-25.

19 Gebretekle GB, Haile Mariam D, Abebe W, et al. Opportunities and barriers to implementing antibiotic stewardship in low and middleincome countries: lessons from a mixed-methods study in a tertiary care hospital in Ethiopia. PLoS One 2018;13:e0208447.

20 Tadesse BT, Ashley EA, Ongarello S, et al. Antimicrobial resistance in Africa: a systematic review. BMC Infect Dis 2017;17:616.

21 Kanapathipillai R, Malou N, Hopman J, et al. Antibiotic resistance in conflict settings: lessons learned in the middle East. JAC Antimicrob Resist 2019;1.

22 Kariuki S, Keddy KH, Antonio M, et al. Antimicrobial resistance surveillance in Africa: successes, gaps and a roadmap for the future. Afr J Lab Med 2018;7:1-2.

23 Soucheray S. Third burial team attacked in DRC; Ebola infects another health worker. CDIRAP, 2019.

24 Country progress in the implementation of the global action plan on antimicrobial resistance: who, Fao, and OIE global tripartite database. Available: http://who.int/antimicrobial-resistance/globalaction-plan/database/en/

25 Das P, Horton R. Antibiotics: achieving the balance between access and excess. Lancet 2016;387:102-4.

26 UNDP, Human Development Report 2015. Available: http://www. hdr.undp.org/en/countries/profiles/GNB as quoted in FIP - Country Profile. WELCOME TO GUINEA BISSAU. MSF [Accessed Aug 2018]. 